\title{
Cost and Efficiency Analysis of Hierarchical SIGMA
}

\author{
Md. Shohrab Hossain, Mohammed Atiquzzaman \\ School of Computer Science, The University of Oklahoma \\ Norman, OK 73019 \\ Email: \{shohrab, atiq\}@ou.edu
}

\author{
William D. Ivancic \\ NASA Glenn Research Center \\ Cleveland, OH 44135 \\ Email: wivancic@grc.nasa.gov
}

\begin{abstract}
To facilitate seamless Internet connectivity to mobile nodes, we earlier proposed SIGMA, a transport layer mobility management protocol. However, frequent handoff of the mobile nodes produce excessive signaling overhead in the bandwidthlimited air interfaces and cause large handover delay, thereby degrading the performance. Hierarchical mobility management for SIGMA aims at resolving this problem by introducing micromobility agents in the hierarchy. In this paper, we perform a comprehensive cost and efficiency analysis of the key mobility management entities of Hierarchical SIGMA taking into account all possible costs. We present numerical results comparing Hierarchical SIGMA with standard hierarchical mobility protocol. Our results demonstrate that Hierarchical SIGMA requires less cost and yields better performance. Our analysis can be used as a framework to compare performance and signaling overheads of mobility protocols for next generation networks.

Index Terms-Host mobility, hierarchical mobility management, handover algorithm, multihoming, cost analysis.
\end{abstract}

\section{INTRODUCTION}

With the proliferation of mobile computing, various mobility management protocols have been proposed to ensure continuous Internet connectivity to mobile devices that generates most of the Internet traffic today. These protocols include Mobile IPv6 (MIPv6) [1] and Hierarchical Mobile IPv6 (HMIPv6) [2] proposed by Internet Engineering Task Force (IETF). However, Mobile IPv6-based protocols suffer from a number of drawbacks, such as, inefficient routing path, high handover latency and packet loss, etc. To address these drawbacks, seamless IP-diversity based mobility protocol, SIGMA [3] was proposed and it decouples location management from data transmission to improve its performance.

For high mobility of nodes, location information are sent very frequently to the location manager, resulting in bandwidth wastage and possible network congestion. Moreover, the correspondent node might get stale information about mobile node's current location if the location manager is far away from the mobile node, resulting in possible connection termination. Therefore, it is very important to reduce the delay of time-critical handover process for improved performance. Hierarchical SIGMA (HiSIGMA) [4], an extension of SIGMA, aims at reducing handover delay and signaling overhead in the

The research work reported in this paper was supported by NASA Grant NNX06AE44G. bandwidth-limited air interfaces by introducing a new micromobility agent in the hierarchy.

Total load on the network facilitating mobile computing are influenced by a number of network parameters (such as, network size, mobility rate, traffic rate). Proliferation in mobile computing have resulted in more signaling and (multimedia) data traffic on different mobility management entities, such as, home agent, mobility anchor point, etc. These entities are very resource restricted and their overloading may result in complete outage for the whole system. Hence, it is crucial to perform entity-wise cost evaluation of hierarchical mobility protocol.

There have been earlier attempts for cost analysis [3]-[9] of mobility protocols. Fu et al. [3], [4] analyze the signaling costs of SIGMA and HMIPv6. Xie et al. [5] propose a dynamic scheme that tries to distribute signaling load for Mobile IP and perform its cost analysis. Makaya et al. [6] present performance and cost analysis of IPv6-based mobility protocols. Lee et al. [7] present a comparative cost analysis of HMIPv6 with Proxy MIPv6. However, none of these works [3]-[7] has performed entity-wise cost evaluation which is essential to estimate actual load on these resource-restricted critical components of a mobility protocol.

The main differences of this work are that we have considered all possible costs required for hierarchical mobility management and have presented an entity-wise cost evaluation for the key mobility management entities of HiSIGMA. We have also defined a metric to compute the data transmission efficiency of HiSIGMA.

The objective of this paper is to analyze the total cost (that includes transmission, processing, searching costs) of mobility management entities of HiSIGMA and figure out how those costs are influenced by network size, mobility rate, traffic rate, and data volume.

Our contributions in this work are: (i) developing mathematical models to estimate total costs of various mobility management entities of HiSIGMA: Anchor Zone Server, Home Zone Server, and complete network and (ii) presenting numerical validation of the model and comparing it with that of HMIPv6 [8].

Our results show that mobility management entities of HiSIGMA incurs much less cost than that of HMIPv6 since the data transmission and hierarchical location management are 


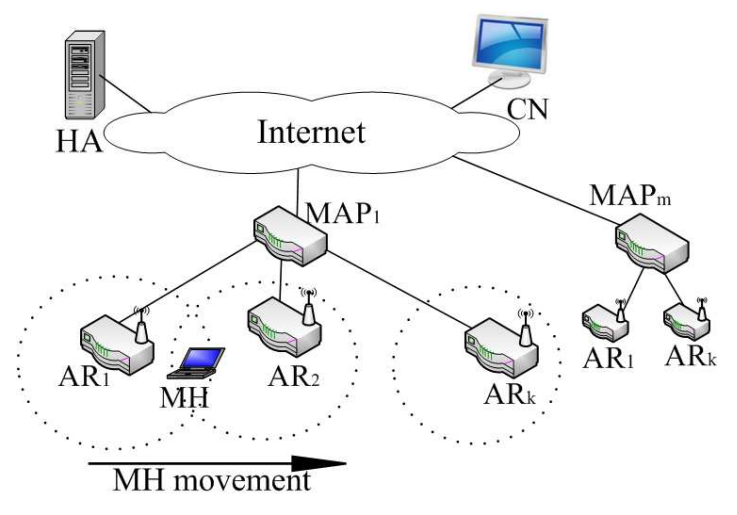

Fig. 1. HMIPv6 Architecture.

decoupled in HiSIGMA unlike HMIPv6. Our analytical cost model can be used as a framework to estimate actual sources (bandwidth, processing power, transmission power) required by key entities of next generation networks.

The rest of the paper is organized as follows. In Section II, HMIPv6 and HiSIGMA protocols are explained in brief. In Section III, analytical models for the cost and efficiency analysis of HiSIGMA are presented. Section IV analyzes the results. Finally, Section V has the concluding remarks.

\section{Hierarchical Mobility management}

In this section, we briefly explain the architecture and operation of standard Hierarchical Mobile IPv6 and our proposed HiSIGMA scheme.

\section{A. Hierarchical Mobile IPv6}

To facilitate micro-mobility in Mobile IPv6 [1], a new mobility agent, called Mobility Anchor Point (MAP) was introduced in the HMIPv6 [2] architecture (Fig. 1) in addition to the Home Agent (HA). Each MAP is essentially a local HA and covers several subnets under its domain. Upon arrival in a new MAP-domain, a Mobile Host (MH) discovers MAP's global address that is stored in the Access Routers (AR) and communicated to the $\mathrm{MH}$ via router advertisements. The $\mathrm{MH}$ then informs its current address (assigned by the MAP) to the HA. All data packets destined to MH are intercepted by the MAP which encapsulates, and forwards them to the MH.

\section{B. Hierarchical SIGMA}

HiSIGMA [4] is based on SIGMA [3] which ensures seamless connectivity between end nodes by sending Binding Update (BU) to the Correspondent Node $(\mathrm{CN})$ and the location manager. This requirement to update location information causes delay and packet loss in active connection, especially when the $\mathrm{MH}$ and its location manager are far away. This situation becomes worse (causing in more delay and packet loss) when the binding updates are required to be authenticated to protect against traffic redirection or man-in-the-middle attacks. Therefore, it is crucial to reduce the delay during time-critical handover process to improve mobility protocol's performance.

In HiSIGMA (Fig. 2), location management is done in multiple levels. A local location manager, namely Anchor

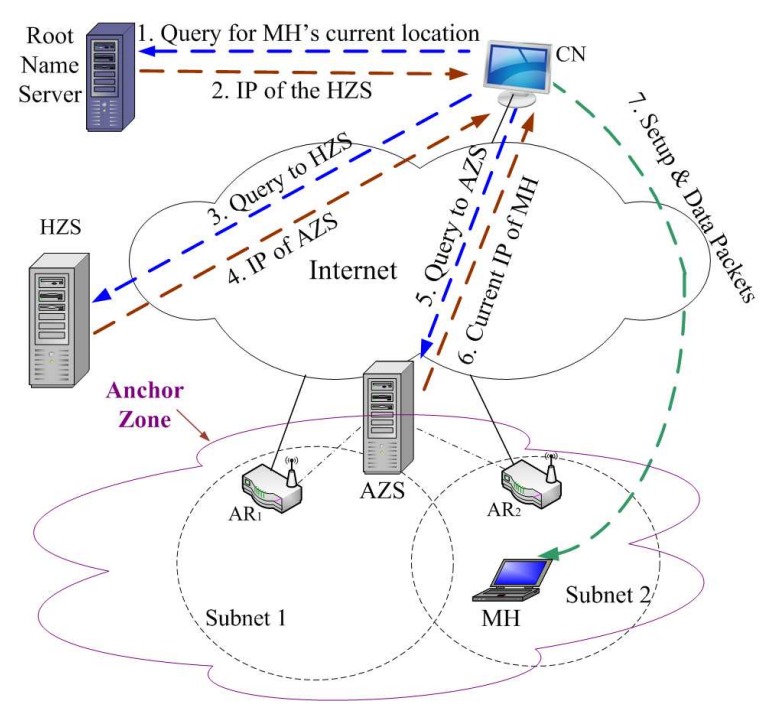

Fig. 2. Hierarchical location management in HiSIGMA.

Zone Server (AZS) is introduced in the hierarchy to keep records of movement within an AZS-domain which covers several subnets. The main difference between this new mobility entity, AZS with HMIPv6's MAP is that AZS does not deal with any data packet destined to the MH since HiSIGMA decouples location management from data transmission. Similar is the case for the Home Zone Server (HZS) which deals with macro-mobility of MHs. When the MH moves within an AZSdomain, there is no need to send signaling traffic outside of the AZS-domain. For movement across AZS-domains, HZS is updated through BU. This hierarchical approach of location management reduces the handover latency and packet loss while improving the accuracy of the location management [4].

It can be noted that DNS can be used as the location manager of SIGMA where domain name is used for the identification of MH. Therefore, location query (for the $\mathrm{MH}$ ) in HiSIGMA can be processed in an approach similar to hierarchical DNS lookup. When $\mathrm{CN}$ wants to setup a new association with $\mathrm{MH}, \mathrm{CN}$ sends a query message (with MH's domain name) to the root name server which replies with HZS's IP address. The CN then queries the HZS which replies with the IP address of current AZS where MH resides. Finally, the $\mathrm{CN}$ queries the AZS which replies with the current IP address(es) of $\mathrm{MH}$.

\section{Analytical Model}

In this section, we present entity-wise cost analysis for HiSIGMA. Specifically, we analyzed total costs for AZS, HZS and the whole network. The network topology, assumptions, notations, cost model and efficiency model are explained in the following subsections.

\section{A. Network Structure}

Fig. 3 shows the two dimensional environment where $x y$ number of ARs (in $x$ rows and $y$ columns) are arranged. There are $m$ AZSs, each of which covers $k$ subnets (let). The HZS is responsible for keeping location information of all the MHs moving in this environment though the number of MHs under 
HZS-domain is assumed to be higher. Each MH communicates with a number of $\mathrm{CNs}$ and each such active communication period is termed as a session.

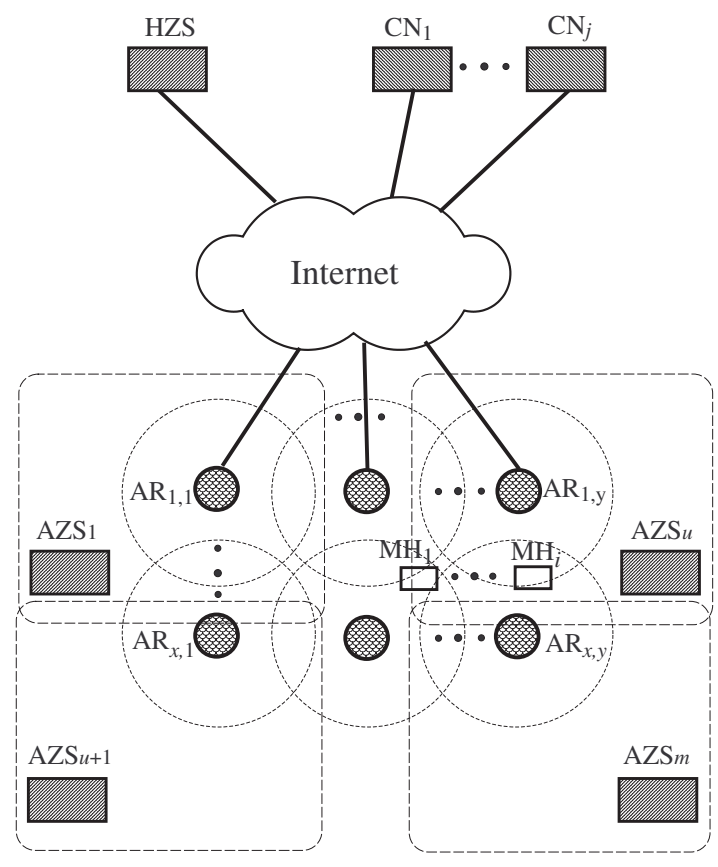

Fig. 3. Network structure for HiSIGMA.

\section{B. Assumptions}

The assumptions of the model are as follows:

- Session arrival rate for each MH is equal.

- Each session length (data size) is equal.

- Uniform distribution of MHs over the whole system.

- Standard IP switching costs are not considered.

\section{Notations}

The notations used in this paper are explained as follows:

$N_{m} \quad$ Number of MHs,

$N_{c} \quad$ Average number of CNs per $\mathrm{MH}$,

$\beta_{X} \quad$ Per hop transmission cost for message type $\mathrm{X}$,

$\Phi_{m h}$ Average distance between MH and HZS (in hops),

$\Phi_{m a}$ Average distance between MH and AZS (in hops),

$\Phi_{h c}$ Average distance between HZS and CN (in hops),

$\Phi_{a c}$ Average distance between AZS and CN (in hops),

$\Phi_{m c}$ Average distance between $\mathrm{MH}$ and $\mathrm{CN}$ (in hops),

$\sigma \quad$ Ratio of overhead wireless link over wired link,

$\eta \quad$ Linear coefficient for lookup cost,

$T_{r} \quad$ Subnet residence time,

$\lambda_{s} \quad$ Average session arrival rate for each mobile host,

$x, y$ Number of access routers in a row or column

$k \quad$ Number of access routers under a AZS,

$m \quad$ Number of AZSs, where $m=x y / k$,

$\kappa \quad$ Maximum transmission unit,

$\alpha \quad$ Average session size,

$\delta_{H} \quad$ Processing cost at HZS,

$\delta_{A} \quad$ Processing cost at AZS.
It should be noted that message type $X$ can be $\{\mathrm{Q}, \mathrm{A}, \mathrm{DP}, \mathrm{DA}$, $\mathrm{RR}, \mathrm{LU}, \mathrm{BU}\}$ which means query, address reconfiguration, data packet, ACK packet, return routability, LU and binding update message.

\section{Cost Model}

In this section, we derive the total cost for AZS, HZS and the whole network for HiSIGMA. Let us first find out the expected number of moves that causes MH's AZS-domain move-out. In the network structure (Fig. 3), there are $x y$ ARs. The MH can move from one AR's coverage area to any other in one move. As each AZS covers $k$ ARs, the probability that the MH will be within the coverage area of the previous AZS (after a movement) is $p=\frac{k}{x y}$. Conversely, the probability that MH will reach a new AZS-domain is $q=1-p=\frac{x y-k}{x y}$. So the probability that the MH moves out of a AZS-domain in $i$ th movement is $P_{i}=p^{i-1} q$. Hence, the expected number of moves for a AZS domain move-out can be obtained as follows:

$$
\begin{aligned}
M & =\sum_{i=1}^{\infty} i P_{i}=q\left(1+2 p+3 p^{2}+4 p^{3}+\ldots\right) \\
& =\frac{1}{1-p}=\frac{x y}{x y-k}
\end{aligned}
$$

1) Anchor Zone Server: As mentioned earlier in Sec II-B, the AZS only deals with location management (not data transmission). The main tasks of AZS are processing 1) query messages from CNs, and 2) registration messages from MHs. The following paragraphs estimates the cost terms of AZS.

a) Query-lookup cost: Each association between $\mathrm{MH}$ and $\mathrm{CN}$ (that happens in every $1 / \lambda_{s} \mathrm{sec}$ ) requires query message and corresponding reply to be exchanged between $\mathrm{CN}$ and AZS (see Fig. 2). On the average, each AZS has $N_{m} / m$ MHs under its domain. So each lookup cost is proportional to $\log _{2}\left(N_{m} / m\right)$. Therefore, cost on AZS for query message is as follows:

$$
\Lambda_{A Z S}^{Q R}=\lambda_{s} N_{c} \frac{N_{m}}{m}\left(2 \beta_{Q}+\eta \log _{2}\left(N_{m} / m\right)\right)
$$

b) Registration cost for micro and macro mobility: In HiSIGMA, an MH does not need to register with the HZS until the MH moves out of the region covered by an AZS, instead it only registers with the AZS. Therefore, every subnet crossing within a AZS (happens every $T_{r}$ seconds) will trigger a registration message (and corresponding ACK) message to (from) the AZS, which incurs transmission cost of $2 \beta_{r a}$ ) and processing cost $\left(\gamma_{a}\right)$ at each AZS. Moreover, in case of the availability of multiple IP addresses, the MH should notify AZS with the primary IP address through dynamic address reconfiguration message which incurs transmission cost of $\beta_{a}$ at AZS. Since all the MHs are uniformly distributed among the m AZS domains, each AZS will have $N_{m} / m$ number of MHs. Therefore, cost at AZS for registration message due to micro-mobility can be obtained as follows:

$$
\Lambda_{A Z S}^{R G 1}=\frac{N_{m}}{m} \times \frac{2 \beta_{L U}+\delta_{A}+2 \beta_{A}}{T_{r}}
$$

For every region crossing between the AZSs, transmission and processing costs are incurred (for registration request-reply messages) at the AZS which is given by, 


$$
\Lambda_{A Z S}^{R G 2}=\frac{N_{m}}{m} \times \frac{2 \beta_{L U}+2 \delta_{A}}{M T_{r}}
$$

c) Total Cost on AZS: Therefore, the total cost on each AZS can be obtained by adding Eqns. (2), (3), and (4):

$$
\Lambda_{A Z S}=\Lambda_{A Z S}^{Q R}+\Lambda_{A Z S}^{R G 1}+\Lambda_{A Z S}^{R G 2}
$$

2) Home Zone Server: In HiSIGMA, the HZS mainly processes 1) query messages from the $\mathrm{CN}, 2$ ) registration messages due to $\mathrm{MH}$ macro-mobility, and 3) return routability test messages.

a) Query-Lookup cost: For each association (that happens in every $1 / \lambda_{s}$ sec) between $\mathrm{MH}$ and $\mathrm{CN}$, query message (and corresponding reply) about the current location of $\mathrm{MH}$ are exchanged between CN and HZS (see Fig. 2). The HZS replies with the IP address of the AZS which replies with the MH's current IP address(es). This incurs transmission cost at the HZS along with looking up a table whose size is proportional to number of MHs it (HZS) covers. Therefore, query-lookup cost on the HZS can be computed as follows:

$$
\Lambda_{H A}^{Q R}=\lambda_{s} N_{m} N_{c}\left(2 \beta_{Q}+\eta \log _{2}\left(c N_{m}\right)\right)
$$

where, c is the ratio of number of MHs under HZS to $N_{m}$.

b) Registration cost for macro-mobility: For the interregion movement of the $\mathrm{MHs}$, transmission and processing costs are incurred at the HZS which is given by,

$$
\Lambda_{H Z S}^{R G}=N_{m} \times \frac{2 \beta_{L U}+\delta_{H}}{M T_{r}}
$$

c) Return routability messages: To avoid session hijacking, RR messages are exchanged among the MH, HZS and CN before $\mathrm{MH}$ sends $\mathrm{BU}$ message to the $\mathrm{CN}$. The Home Test Init (HoTI) and Home Test (HoT) messages are sent through the HZS for RR procedure. This happens for every $M T_{r}$ seconds and for every MH-CN pair under the HA. Therefore, the cost on HZS for RR messages is as follows:

$$
\Lambda_{H Z S}^{R R}=N_{m} N_{c} \times \frac{4 \beta_{R R}}{M T_{r}}
$$

d) Total Cost on HZS: Thus, the total cost on the HZS can be obtained by adding Eqns. (6), (7), and (8):

$$
\Lambda_{H Z S}=\Lambda_{H Z S}^{Q R}+\Lambda_{H Z S}^{R G}+\Lambda_{H Z S}^{R R}
$$

3) Complete Network: To compute the total cost on the whole system, we will consider resources consumed due to HiSIGMA protocol. These include transmission, lookup and processing cost incurred in the whole network along the route of various mobility protocol messages as well as the data packets. These are explained in the following paragraphs.

a) Query-lookup cost: The transmission cost for all the query and reply messages towards the HZS is $N_{c} N_{m}\left(2 \Phi_{h c} \beta_{q}\right) \lambda_{s}$. Per entry searching cost at HZS is $\left.\eta \lambda_{s} \log c N_{m}\right)$. Similar estimation can be done $\mathrm{m}$ AZSs of the network structure. Hence, the cost of the network for the query messages is give by,

$\Lambda_{N e t}^{Q R}=N_{m} N_{c} \lambda_{s}\left(2 \beta_{q} \Phi_{h c}+\eta \log _{2}\left(c N_{m}\right)+2 \beta_{q} \Phi_{a c}+\eta \log _{2}\left(N_{m} / m\right)\right)$ b) Registration costs for macro and micro mobility: As the MH only require to register with the HZS only if it moves out of the region covered by an AZS. Otherwise, in every $T_{r}$ sec, $\mathrm{MH}$ registers with the AZS which is $\phi_{m a}$ hops away that includes one wireless hop. MHs also exchange address reconfiguration messages with the AZS during this micro-mobility. Due to frame retransmissions and medium access contentions at the data link layer of wireless links, transmission cost of a wireless hop is higher than that of a wired hop; we denote this effect by a proportionality constant, $\sigma$. Hence, the cost incurred at the network for MH's micromobility is as follows:

$$
\Lambda_{N e t}^{R G 1}=N_{m} \times \frac{2\left(\beta_{L U}+\beta_{A}\right)\left(\phi_{m a}-1+\sigma\right)+\delta_{A}}{T_{r}}
$$

On the other hand, for every region crossing (in every $M T_{r}$ sec), $\mathrm{MH}$ registers with the HZS which is $\phi_{m h}$ hops (that includes one wireless hop) away from the MH. This incurs transmission cost, processing cost at the HZS, and processing cost at AZS(s). Since there is only one location update per subnet crossing, no matter how many $\mathrm{CNs}$ an $\mathrm{MH}$ is communicating with, the number of $\mathrm{CNs}$ does not have any impact on the location update cost. Therefore, the cost at incurred at the network for the macro-mobility of all the MHs can be obtained as follows:

$$
\begin{aligned}
\Lambda_{N e t}^{R G 2} & =N_{m} \frac{2 \beta_{L U}\left(\Phi_{m h}-1+\sigma\right)+\delta_{H}}{M T_{r}} \\
& +N_{m} \frac{2 \beta_{L U}\left(\Phi_{m a}-1+\sigma\right)+2 \delta_{A}}{M T_{r}}
\end{aligned}
$$

c) Return routability messages: The RR messages are sent every $M T_{r}$ sec by the $\mathrm{MH}$ to HZS which forwards them to $\mathrm{CN}$. The HoTI message follow the path between $\mathrm{MH}$ and HZS (which is of $\phi_{m h}$ hops with one wireless hop) and the path between HZS and CN of $\phi_{h c}$ wired hops. Each HoT message incurs similar cost. Each CoTI message is sent directly to $\mathrm{CN}$ from the $\mathrm{MH}$ which uses $\phi_{m c}$ hops (that includes one wireless hop). Therefore, cost on the network for RR messages are:

$$
\Lambda_{N e t}^{R R}=\frac{N_{m} N_{c}}{M T_{r}} 2 \beta_{r r}\left(\phi_{m h}+\phi_{h c}+\phi_{m c}-2+2 \sigma\right)
$$

d) Binding update cost: For each $\mathrm{CN}$ communicating with an $\mathrm{MH}$, the $\mathrm{MH}$ needs to send a binding update (with a transmission cost $\beta_{B U}$ ) after each handover. Therefore, the binding update cost per second in the whole system can be calculated by multiplying the number of $\mathrm{MHs}$, the average number of communicating $\mathrm{CNs}$, and the average cost per binding update:

$$
\Lambda_{N e t}^{B U}=N_{m} N_{c} \frac{2\left(\Phi_{m c}-1+\sigma\right) \beta_{B U}}{T_{r}}
$$

e) Packet delivery cost: Similar to the analysis in [5], we have considered data packet transmission cost while estimating the total cost on the whole system. HiSIGMA is free of packet encapsulation or decapsulation. Packets from $\mathrm{CN}$ to $\mathrm{MH}$ follows direct route with $\Phi_{m c}$ hops including one wireless hop. As the average session length is $\alpha$, and maximum transmission unit is $\kappa$, there are $\left\lceil\frac{\alpha}{\kappa}\right\rceil$ number of packets, and the packet rate can be obtained by $\left\lceil\frac{\alpha}{\kappa}\right\rceil \times \lambda_{s}$. The transmission cost for data 


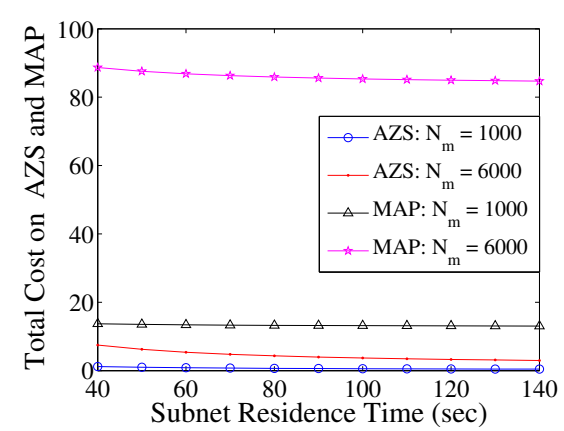

Fig. 4. Total cost at each AZS and MAP as functions of subnet residence time.

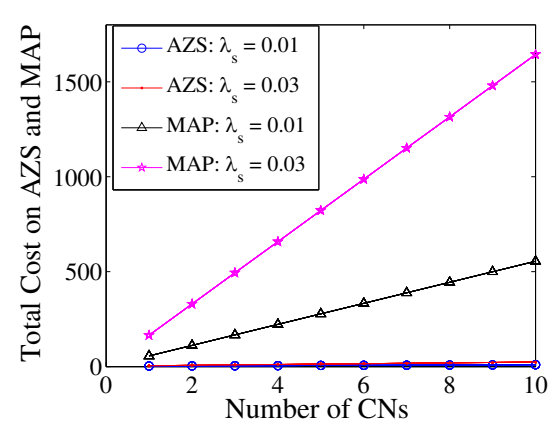

Fig. 5. Total cost at each AZS and MAP as functions of number of $\mathrm{CNs}$.

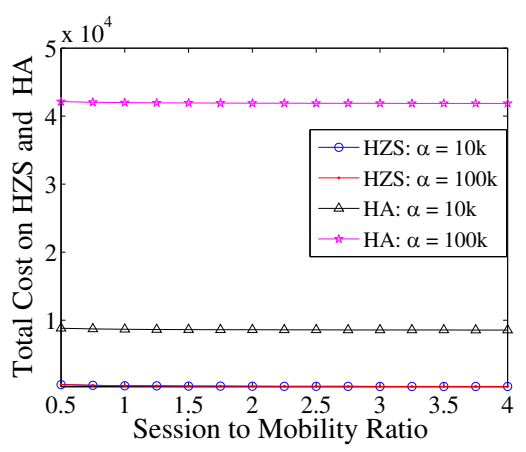

Fig. 6. Impact of SMR on total cost of HZS and HA for different session lengths. packet and corresponding acknowledgement is $\left(\beta_{D P}+\beta_{D A}\right)$. Thus, the packet delivery cost of the whole network can be expressed as:

$$
\Lambda_{N e t}^{P D}=N_{m} N_{c} \lambda_{s}\left\lceil\frac{\alpha}{\kappa}\right\rceil\left(\beta_{D P}+\beta_{D A}\right)\left(\Phi_{m c}-1+\sigma\right)
$$

f) Total cost of the Network: The total on the network due to HiSIGMA protocol can be obtained by adding Eqns. (10), (11), (12), (13), (14), and (15) as:

$$
\Lambda_{N e t}=\Lambda_{N e t}^{Q R}+\Lambda_{N e t}^{R G 1}+\Lambda_{N e t}^{R G 2}+\Lambda_{N e t}^{R R}+\Lambda_{N e t}^{B U}+\Lambda_{N e t}^{P D}
$$

\section{E. Efficiency}

We define a new metric called efficiency to measure the performance of mobility management protocols. It is defined as the ratio of net data delivery cost (excluding all overheads along the optimal route) to the total cost (that includes signaling and data delivery costs) required for the mobility protocol. Since HiSIGMA uses direct (optimal) route between $\mathrm{MH}$ and $\mathrm{CN}$, Eqn. (15) gives the net data delivery cost for the whole system. Therefore, the efficiency HiSIGMA protocol can be computed as follows:

$$
\xi^{\text {HiSIGMA }}=\frac{\Lambda_{\text {Net }}^{P D}}{\Lambda_{N e t}}
$$

\section{NUMERICAL RESUltS}

In this section, we present results showing impact of various system parameters on HiSIGMA and its mobility management entities. A similar cost analysis for Hierarchical Mobile IPv6 has been performed in [8]. The AZS and HZS of HiSIGMA corresponds to the MAP and HA of HMIPv6, respectively. We compare HiSIGMA results with HMIPv6.

The parameter values used in numerical analysis are derived using similar approaches used in [3], [5], [10]; each cost metric is a relative quantity and is based on the specific packet size (unit cost for 100 bytes [5], [10]). For example, if a signaling packet is 60 bytes long, the corresponding transmission cost is 0.6. Therefore, we have set the parameters as follows: $\beta_{Q}$ $=0.60, \beta_{A}=1.40, \beta_{D P}=5.72, \beta_{D A}=0.60, \beta_{R R}=0.60$, $\beta_{L U}=0.60, \beta_{B U}=0.60, \sigma=10, \eta=0.3, c=10, T_{r}=70$ sec, $\eta=0.3, \lambda_{s}=0.01, \delta_{H}=0.3, \delta_{A}=0.3, \kappa=512 \mathrm{bit} / \mathrm{sec}$, $\alpha=10240$ bits. The default values of other parameters are $x$ = 51, $y=34, k=12, N_{c}=1, N_{m}=4000$. The number of hops between mobility entities is assumed to be 10 which is reasonable for networks within the US. So we have set $\Phi_{m h}$ $=\Phi_{h c}=\Phi_{m c}=\Phi_{a c}=10$. The hop count between the $\mathrm{MH}$ and AZS is assumed to be 2 (i.e., $\Phi_{m a}=2$ ).

In order to obtain the general trend / impact of different network parameters, we have varied their values in a wide range. For example, we varied number of MHs between 2,000 to 7,000; number of $\mathrm{CNs}$ per $\mathrm{MH}$ between 1 to 10 ; session arrival rate between 0.01 to 0.1 ; average session size between $10 \mathrm{~Kb}$ to $100 \mathrm{~Kb}$; Session to Mobility Ratio (SMR) between 0.50 to 4 (SMR is defined as $\left.T_{r} \times \lambda_{s}\right)$.

\section{A. AZS vs. MAP}

Fig. 4 shows the impact of subnet residence time $\left(T_{r}\right)$ on the total cost of each AZS (HiSIGMA) and MAP (HMIPv6). The total cost on each AZS is found to be much less than that for each MAP. This is because data transmission in HiSIGMA is decoupled from hierarchical location management and there is no tunneling or encapsulation of data packets through the AZS in HiSIGMA (unlike HMIPv6). From Fig. 4, we also find that total cost reduces for higher values of $T_{r}$ which implies slower speed of MHs. Slow speed of MHs causes less number of handoffs, thereby reducing signaling costs, e.g., registration, BU, RR costs.

In Fig. 5, the total costs of each AZS and MAP are shown as functions of number of CNs. Higher number of CNs generates more traffic, thereby increasing the load on the MAP, unlike for AZS which does not deal with data traffic. Hence, the cost for AZS is very low compared to MAP.

\section{B. $H Z S$ vs. $H A$}

The impact of SMR on total cost of HZS and HA is shown in Fig. 6 for different session lengths. We kept $\lambda_{s}$ fixed to 0.01 while varying $T_{r}$ from 50 to $400 \mathrm{sec}$, which yields a SMR of 0.50 to 4 . Higher session lengths produces more data; hence, data delivery cost increases compared to the signaling traffic. This increases the total cost of HA. However, the total cost of HZS is not affected much by higher session length since HZS has nothing to do with data packets in HiSIGMA.

Fig. 7 shows the impact of number of CNs on the total cost of HZS and the HA for different session arrival rates. Again, the total cost of HA is much higher than that of HZS due to the tunneling / encapsulation of data packets between 


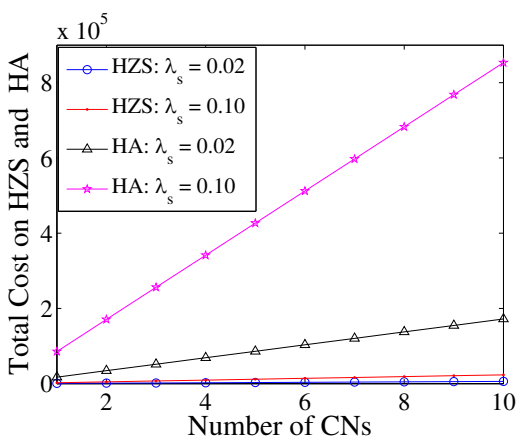

Fig. 7. Impact of number of $\mathrm{CNs}$ on total cost of HZS and HA for different session arrival rates.

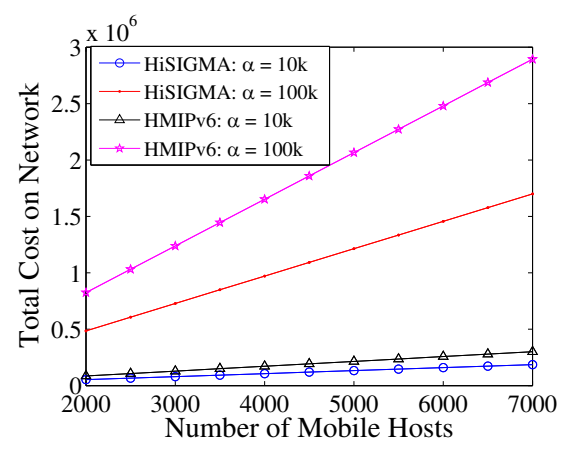

Fig. 8. Total cost on the network as functions of number of MHs.

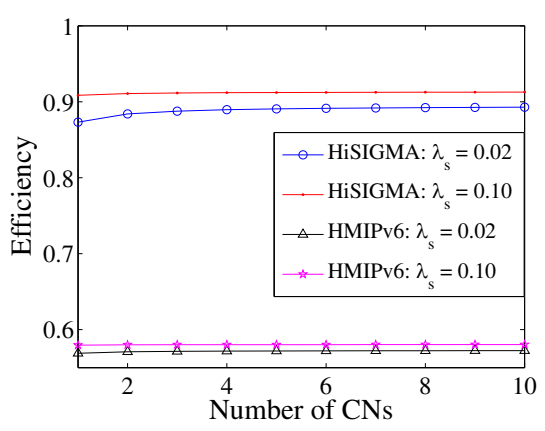

Fig. 9. Efficiency of the whole system vs. number of CNs.

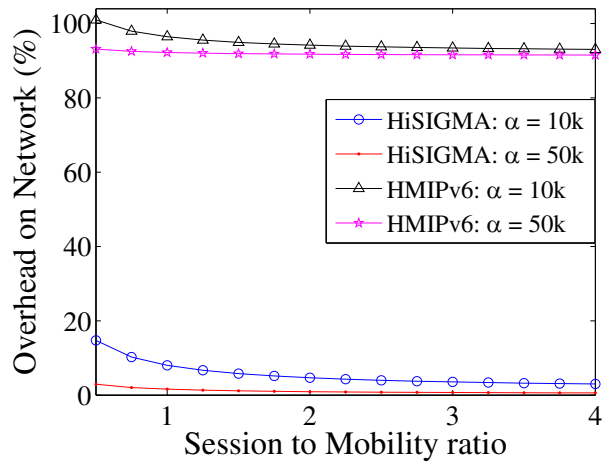

Fig. 10. Percentage overhead of the whole system vs. SMR.

MH-CN pairs unlike in HiSIGMA. However, the total cost of HZS slowly increases for higher values of $N_{c}$ due to higher signaling costs, e.g., query-lookup, registration and RR cost.

\section{Complete Network}

In Fig. 8, the impact of number of MHs on the total cost of the whole system is shown varying session lengths. Higher number of MHs increases the total cost in all cases. However, the rate of increase is much higher for HMIPv6 protocol due to additional overhead of packet tunneling through the HA, and use of non-optimal route between $\mathrm{MH}-\mathrm{CN}$ pairs.

Fig. 9 shows the impact of number of $\mathrm{CNs}$ and session arrival rate on the efficiency of HiSIGMA and HMIPv6 protocols. It is found that HiSIGMA exhibits much higher efficiency than HMIPv6. Moreover, higher value of $\lambda_{s}$ causes more data traffic into the system, thereby raising the efficiency values of both protocols.

Finally, Fig. 10 shows the impact of SMR on the percentage overhead on the whole system per unit data traffic of HiSIGMA and HMIPv6 protocols. The overhead on HMIPv6 system is found to be much higher than HiSIGMA due to the use of suboptimal path and tunneling.

\section{Summary of Results}

In summary, we find that the total costs of mobility management entities of HiSIGMA are much less than that of HA and MAP (HMIPv6), respectively. This is because data transmission and location management are decoupled in HiSIGMA unlike HMIPv6. In addition, the efficiency of HiSIGMA is much higher than HMIPv6 since HiSIGMA lacks any encapsulation and tunneling of data packets and uses direct route between $\mathrm{MH}$ and $\mathrm{CN}$.

\section{CONClusion}

In this paper, we have developed an analytical model to perform entity-wise cost evaluation for the mobility management entities of Hierarchical SIGMA, a transport layer mobility protocol. We have compared signaling overhead of HiSIGMA entities with that of HMIPv6 in terms of cost and efficiency. Results show that Hierarchical SIGMA incurs less costs on its key entities and yields better efficiency than HMIPv6. The cost and efficiency analysis presented in this paper will help network engineers in estimating actual resource requirements for the key entities of next generation networks.

\section{REFERENCES}

[1] D. Johnson, C. E. Perkins, and J. Arkko, "Mobility support in IPv6," IETF RFC 3775, Jun 2004.

[2] H. Soliman, C. Castelluccia, K. E. Malki, and L. Bellier, "Hierarchical Mobile IPv6 mobility management (HMIPv6)," IETF RFC 5380, Oct 2008.

[3] S. Fu and M. Atiquzzaman, "Signaling cost and performance of SIGMA: A seamless handover scheme for data networks," Wireless Communication and Mobile Computing, vol. 5, no. 7, pp. 825-845, Nov 2005.

[4] - "Hierarchical location management for transport layer mobility," in IEEE GLOBECOM, San Francisco, CA, Nov 27-Dec 1, 2006.

[5] J. Xie and I. Akyildiz, "A novel distributed dynamic location management scheme for minimizing signaling costs in Mobile IP," IEEE Transactions on Mobile Computing, vol. 1, no. 3, pp. 163-175, Jul 2002.

[6] C. Makaya and S. Pierre, "An analytical framework for performance evaluation of IPv6-based mobility management protocols," IEEE Transactions on Wireless Communications, vol. 7, no. 3, pp. 972-983, Mar 2008.

[7] J.-H. Lee, T.-M. Chung, and S. Gundavelli, "A comparative performance analysis on Hierarchical Mobile IPv6 and Proxy Mobile IPv6," in IEEE PIMRC, Poznan, Poland, Sep 15-18, 2008.

[8] M. S. Hossain, M. Atiquzzaman, and W. Ivancic, "Cost analysis of mobility entities of Hierarchical Mobile IPv6," in IEEE MILCOM, San Jose, CA, Oct 31-Nov 3, 2010.

[9] - "Cost analysis of mobility management entities for sigma," in High Performance Switching and Routing (HPSR), Richardson, TX, Jun 13-16, 2010.

[10] J. Xie and U. Narayanan, "Performance analysis of mobility support in IPv4/IPv6 mixed wireless networks," IEEE Transactions on Vehicular Technology, vol. 59, no. 2, Feb 2010. 